“(C) 2015 IEEE. Personal use of this material is permitted. Permission from IEEE must be obtained for all other uses, in any current or future media, including reprinting/republishing this material for advertising or promotional purposes, creating new collective works, for resale or redistribution to servers or lists, or reuse of any copyrighted component of this work in other works." 


\title{
Energy-Delay Tradeoffs in Impulse-based Ultra-Wideband Body Area Networks with Noncoherent Receivers
}

\author{
Mohammad Sadegh Mohammadi* $^{*}$, Qi Zhang*, Eryk Dutkiewicz ${ }^{\dagger}$, Xiaojing Huang $^{\ddagger}$ and Rein Vesilo ${ }^{\dagger}$ \\ *Department of Engineering, Aarhus University, Denmark \\ Email: $\{$ msmo, qz $\} @$ eng.au.dk \\ ${ }^{\dagger}$ Engineering Department, Macquarie University, Australia \\ Email: \{eryk.dutkiewicz, rein.vesilo\}@mq.edu.au \\ $\ddagger$ Computational Informatics, CSIRO, Australia \\ Email: xiaojing.huang@csiro.au
}

\begin{abstract}
In this paper we address the problem of rate scheduling in the Impulse Radio (IR) ultra-wideband (UWB) wireless body area networks (WBANs) and the minimum energy required to stabilize the queuing system. Targeting low complexity WBAN applications, we assume noncoherent receivers based on energy detection and autocorrelation for all nodes. The coordinating node can minimize the average energy consumption of the system and achieve the queue backlog stability of the sensor nodes by controlling the number of pulses per symbol. We first illustrate the necessary and sufficient conditions of network stability for a multi-mode UWB system and then propose a feasible rate scheduling algorithm based on the Lyapunov optimization theory. The scheduling algorithm uses the instantaneous channel state information and the length of the local queue of all sensor nodes and can approach the optimal energy-delay tradeoff of the network. We apply our theoretical framework to the IRUWB physical layer of the IEEE 802.15.6 standard and extract the optimal physical layer modes that can achieve the desired energy-delay tradeoff.
\end{abstract}

\section{INTRODUCTION}

WBAN is one of the promising technologies that can enable low-cost and ubiquitous health care solutions by means of tiny wearable or implantable sensors. Energy efficiency and reliability are two crucial issues of WBANs that have attracted extensive research efforts during the past few years. To enable ubiquitous and unobstructive health care solutions, wireless links are preferred. However, the wireless channel in typical wearable and implant WBANs is highly unstable due to the proximity to the body and the unknown dispersive characteristics of different tissues as well as the shadowing and reflective effects from a set of random parameters such as the clothings or possibly, medical implants that vary person by person. On the other hand, the vital importance of the physiological information calls for reliable wireless communication in WBANs. Such a solution is also required to have a low-power implementation. The reason lies in that 1) to avoid tissue heating problem; 2) the battery becomes even smaller in WBAN due to the small form factor of the sensor nodes to meet the non-invasive requirements. To achieve reliable communication in WBANs, it could consume the major portion of the battery energy [1]. Based on this fact, energy efficient design of the transmission protocol, which has a remarkable role in reliability, is a major step to achieve an optimal design.
Indeed, the transmission protocol should be able to allocate optimum transmission energy based on the current state of the system to fulfill reliable communication.

For delay sensitive traffic in WBAN applications, the transmission protocol should take into account the data queuing delay to meet quality of service (QoS) requirements. In the study of delay sensitive communications over wireless networks, the cross-layer approaches combine the physical layer (PHY) models with the queue theory to optimize the spectrum and energy resource allocation based on the tradeoff between radio resource utilization and the average delay [2]-[5]. Moreover, it is aiming to adjust the scheduling policy to achieve the desired energy-delay tradeoff with an energy expenditure arbitrarily close to the optimal. We consider IR-UWB networks with noncoherent receivers based on energy detection (ED) and autocorrelation (AC) since this setup is an ideal combination of the PHY technologies that can provide unique capabilities such as low complexity and precision localization together with low implementation cost. We assume the transmission rate (e.g. the transmission PHY mode) can be adapted by an adaptive modulation and coding scheme that controls the number of pulses per symbol. Our study closely follows the theoretical framework in [2]-[4]. Based on the observed instantaneous channel state information (CSI) and queue length information (QLI), the rate scheduling policy is able to select a PHY mode with minimum transmission energy for each sensor node while maintaining the network stability.

The contribution of this paper is to extend the existing theoretical frameworks in the study of delay-sensitive data transmission to the IR-UWB WBANs with noncoherent receivers. We formulate the problem using our PHY model and apply the results to the IEEE 802.15.6 based IR-UWB WBANs. We first calculate the minimum energy function, i.e., the minimum required energy that is required to achieve the network stability for a given traffic rate and provide the mathematical representation for the stability constraint. Then, we identify the optimal energy-delay tradeoff in the system which is fundamental in the design of the scheduling algorithm and expresses the minimum required energy to achieve a specific average delay. Therefore, we pursue a cross-layer approach to integrate the PHY models of the IR-UWB WBANs 
with the existing higher layer scheduling policies proposed in [2] and [3] to come up with an explicit design model for these networks. Furthermore, we include the total consumed energy required for sensing, processing and communication in the optimization problem for a more comprehensive evaluation, despite [2]-[4] that only consider the transmission energy in their scheduling algorithm.

The paper is organized as follows. Section II characterizes the PHY model of the IR-UWB WBANs and the overall network setup. Based on the presented PHY model, we formulate the minimum-energy rate scheduling problem in the form of an optimization problem and derive the necessary and sufficient conditions for network stability in Section III. In this section we also propose a rate scheduling algorithm based on the instantaneous CSI and the average arrival rate at the given sensor that can stabilize the buffers with minimum energy. In Section IV, we describe the scheduling algorithm based on the CSI and QLI of all sensor nodes that can approach the optimal energy-delay tradeoff of the system. We apply the derived theoretical results to the IEEE 802.15.6 based IR-UWB WBANs in Section $\mathrm{V}$ and identify the optimal energy-delay tradeoffs for different traffic scenarios. We also simulate and validate the operation of the proposed rate scheduling algorithm for the IEEE 802.15.6 channel model assuming different parameters. The conclusions are pointed out in Section VI.

\section{SySTEM MODEL}

\section{A. Physical Layer Characteristics}

We assume a WBAN of $N_{s}$ wireless sensor nodes (motes) and a master hub node that communicate using noncoherent IR-UWB transceivers. To enable various data rates, every node can transmit with a specific modulation and coding scheme which we refer to as a physical layer mode. This can normally be achieved by changing the number of pulses per symbol in IR-UWB systems. Explicitly, considering there are $N_{m}$ PHY modes and a given physical layer mode $m \in\left[0, N_{m}\right]$, each sensor node uses $N_{p}^{(m)} \in \mathbb{Z}_{+}$pulses of the form $p(t)$ with duration $T_{p}$ and energy $E_{p}$ to transmit a symbol. The transmitted waveform is represented as

$$
x_{k}^{(m)}(t)=\sum_{i} w^{(m)}\left(t-c_{i} T_{w}^{(m)}-i T_{s}^{(m)}\right),
$$

where $k \in\left[1, \ldots, N_{s}\right]$ and

$$
w^{(m)}(t)=\sum_{j=0}^{N_{p}^{(m)}-1} p\left(t-j T_{p}\right),
$$

is a waveform of duration $T_{w}^{(m)}=N_{p}^{(m)} T_{p}$, and $c_{i}$ is the time hopping coefficient selected from the range $\left[0, N_{w}-1\right] . N_{w}$ indicates the number of possible waveform positions within a symbol time. The symbol time

$$
T_{s}^{(m)}=N_{w} T_{w}^{(m)},
$$

changes over different PHY modes and hence the amount of transmitted data within a time unit varies depending on the PHY mode. We denote the total energy consumption corresponding to PHY mode $m$ by

$$
E_{T}^{(m)}=N_{p}^{(m)} E_{T P P},
$$

where $E_{T P P} \triangleq \alpha_{t} E_{t}+\alpha_{r} E_{r}$ is the total weighted energy per pulse consisting of the transmission and reception energies $E_{t}$ and $E_{r}$ respectively. $\alpha_{t}$ and $\alpha_{r}$ are the energy weights at the transmitter and the receiver, $E_{t}$ is sum of the pulse energy $E_{p}$ and the total sensing and processing energies at the transmitter averaged per pulse. Generally, the UWB impulses are subject to regulatory spectral masks and therefore the transmitted pulse energy $E_{p}$ is usually selected such that $E_{p} \leq E_{\max }$, where $E_{\max }$ is the maximum pulse energy permissible by the regulatory. Therefore, to increase the probability of pulse detection, it is useful to assume a fixed $E_{p}=E_{\max }$. The received signal at the hub is given by

$$
y_{k}^{(m)}(t)=\sum_{i} x^{(m)}(t) * h_{k}(t)+n(t),
$$

where $*$ denotes linear convolution, $n(t)$ is a zero-mean additive white Gaussian noise process with two-sided power spectral density $N_{0} / 2$, and $h_{k}(t)$ is the normalized multipath channel for the $k$ 'th sensor with $N_{m p, k}$ resolvable multipath components given by

$$
h_{k}(t)=\sum_{l=0}^{N_{m p, k}-1} a_{k, l} \delta\left(t-\tau_{k, l}\right),
$$

in which $\left\{a_{k, l}\right\}$ and $\left\{\tau_{k, l}\right\}$ represent the set of corresponding resolvable multipath coefficients and delays, respectively.

We define the average transmission rate corresponding to PHY mode $m$ as

$$
r^{(m)}=\frac{1}{N_{p}^{(m)}}, m \in\left[1, . ., N_{m}\right],
$$

which is the fractional bit per pulse assuming one bit per symbol. Obviously, the maximum possible rate is achieved by using one pulse per symbol. We define $r^{(0)}=N_{p}^{(0)}=0$, which represents the idle mode where the transmission rate and energy are zero. Without loss of generality we assume $0<r^{(1)}<r^{(2)}<\cdots<r^{\left(N_{m}\right)}$.

We consider noncoherent reception including ED and $\mathrm{AC}$ schemes. ED can be combined with either on-off keying (OOK) or pulse position modulation (PPM). AC is usually based on either transmitted reference or differential signaling. The energy detectors can detect the existence of a signal in a specific time duration by integrating the square of the incoming signal. Similarly, the autocorrelation receivers integrate the product of the input signal and a noisy template over a specific time duration. In any case, the desired fraction of the total collected energy is strongly affected by the integration time $T_{i}$ and the state of the wireless channel. Let $T_{p}=2.003$ ns (see [7]) and assume the integration window covers the entire timeslot such that $T_{i}^{(m)}=T_{w}^{(m)}=N_{p}^{(m)} T_{p}$. Therefore, intra symbol interference can occur within a timeslot when the channel's RMS delay spread is large enough compared with $T_{p}$. Explicitly, the Gaussian approximation for the bit error probability of the ED-binary PPM for the PHY mode $m$ is given in [6] as

$$
P_{b} \approx Q\left(\frac{\bar{\mu}_{k} N_{p} E_{p} / N_{0}}{\sqrt{2 \bar{\mu}_{k} N_{p} E_{p} / N_{0}+2 N_{p} B T_{p}}}\right),
$$

where $Q(\cdot)$ is the tail probability of the standard normal distribution, $B$ denotes bandwidth, $\bar{\mu}_{k}=\mathbb{E}\left\{\mu_{k}\right\}$ in which $\mathbb{E}[\cdot]$ 


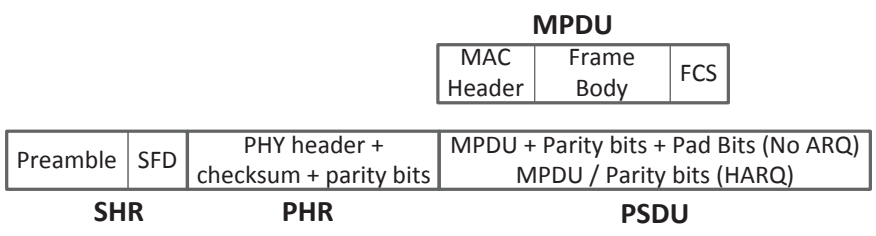

Fig. 1. IEEE 802.15.6 UWB PHY packet structure [7].

is expectation operation, $\mu_{k}=\sum_{l=0}^{N_{p}-1} \mu\left(T_{w}-l T_{p} \mid h_{k}\right) / N_{p}$ and $\mu_{k}$ is the multipath energy capture index for the received signal from the $k^{\prime}$ 'th sensor. This parameter is in general, a function of the integration time $T$ and the multipath channel $h$ and is defined as [6]

$$
\mu(T \mid h)=\int_{t=0}^{T}|h(t)|^{2} d t, \mu(T \mid h) \in[0,1] \forall k .
$$

It follows that the performance of the transmitted reference signaling can be given by (8) too. Also in case of differential signaling, we can use this equation but it performs the same as with half of the signal's energy since it is a transmitted reference system that saves the reference pulses. We define the instantaneously received SNR as $\gamma_{k}=\mu_{k} E_{p} / N_{0}$. Hence, the average received SNR can be expressed by

$$
\bar{\gamma}_{k}=\bar{\mu}_{k} E_{p} / N_{0},
$$

In the sequel, we pursue a cross-layer approach to design a rate scheduling policy that is influenced by the physical layer success probabilities.

\section{B. Network Model}

The medium access of each sensor node is controlled by the hub with full knowledge of the received SNR from all the sensor nodes. Each sensor has a buffer that stores the arrived data from the application layer in a service queue. The network operates in slotted time $t$ with time slot duration $\Delta t$. In each beacon period the hub uses management/control packets to inform the sensors about their scheduled physical layer parameters, i.e. the corresponding sensor node and the PHY mode to transmit. For a given time slot $t$, we denote the scheduled sensor by $K(t)$ and the allocated mode by $M_{k}(t)$ respectively. Each data packet consists of a synchronization header (SHR), a physical layer header (PHR), and the corresponding physical layer service data unit (PSDU). The SHR and the PHR constitute the physical layer overhead bits and enable the receiver to correctly detect the packet and acquire the physical layer settings such as the packet length and the PHY mode. For the PHY mode $m$ and sensor $k$, let $P_{S H R, k}^{(m)}$, $P_{P H R, k}^{(m)}$, and $P_{P S D U, k}^{(m)}$ denote the probability of successful reception of the SHR, PHR, and PSDU at the hub, respectively. The packet success rate (PSR) is expressed as

$$
\mathrm{PSR}_{k}^{(m)}=P_{S H R, k}^{(m)} P_{P H R, k}^{(m)} P_{P S D U, k}^{(m)} .
$$

We assume that the channel state remains constant during the transmission of one data packet.

\section{Minimum Energy with Stability CONSTRAint AND CHANNEL STATE INFORMATION}

Let $A_{k}(t)$ represent an i.i.d. ergodic bit arrival process over time slots corresponding to the $k$ 'th sensor node with the arrival rate $\mathbb{E}\left[A_{k}(t)\right]=\lambda_{k}$ bits per time slot. We assume that the arrival process is independent of the queue backlog and is bounded, i.e. there exists $A_{\max }$ such that $A_{k}(t) \leq$ $A_{\text {max }}, \forall t, k$. For each sensor node $s_{k}$ we denote the state of the queue backlog by $Q_{k}(t)$ over time. The queue dynamics can be modeled by the following equation

$$
Q_{k}(t+1)=\max \left\{Q_{k}(t)-F_{k}(t), 0\right\}+A_{k}(t),
$$

where $F_{k}(t)$ is the finished work at time $t$, i.e. the transmitted data from the sensor. We also assume $\lambda_{k} \in \Lambda, \forall k \in\left[1, N_{s}\right]$, where $\Lambda$ is the set of all arrival rates for which there exists a randomized scheduling policy that can achieve a finite average delay (Similarly, we denote the complement region of $\Lambda$ by $\bar{\Lambda}$ ). Such a policy is defined for any time slot $t$ by the conditional probability distribution

$$
P_{K, M \mid \Gamma}=\mathbb{P}[K=k, M=m \mid \boldsymbol{\Gamma}=\boldsymbol{\gamma}],
$$

where $K$ and $M$ are random variables over $\left\{1, \ldots, N_{s}\right\}$ and $\left\{1, \ldots, N_{m}\right\}$ indicating the sensor index and the scheduled PHY mode, and $\boldsymbol{\Gamma}$ is a random vector over the channel state vector $\gamma=\left(\gamma_{1}(t), \ldots, \gamma_{N_{s}}(t)\right)^{\mathrm{T}}$.

The probability distribution in (13) can be written in matrix form $\mathbf{W}(\gamma)$ of size $N_{s} \times\left(N_{m}+1\right)$ with elements $w_{k, m}(\gamma) \in$ $[0,1]$ such that

$$
w_{k, m}(\boldsymbol{\gamma})=\mathbb{P}[K=k, M=m \mid \boldsymbol{\Gamma}=\boldsymbol{\gamma}] .
$$

To define the stability of the queue $Q_{k}(t)$, we define $q_{k}(U)$ as the largest limiting fraction of time the queue backlog is above $U$ [3], i.e.

$$
q_{k}(U)=\limsup _{t \rightarrow \infty} \frac{1}{t} \sum_{t^{\prime}=0}^{t} \mathbb{P}\left\{Q_{k}\left(t^{\prime}\right)>U\right\},
$$

then the queue is stable if

$$
\lim _{U \rightarrow \infty} q_{k}(U)=0,
$$

where limsup denotes the limit superior. It follows that the necessary and sufficient condition for the stability of the queue for each sensor $k$ is [3]

$$
\mathbb{E}\left[F_{k}(t)\right] \geq \lambda_{k},
$$

which necessitate that the average transmission rate for each sensor should be at least equal to its arrival rate ${ }^{1}$. The optimal rate scheduling policy is the solution of the following optimization problem

$$
\begin{array}{ll}
\text { Minimize: } & \mathbb{E}\left[\sum_{k} \sum_{m} w_{k, m}(\gamma) E_{T}^{(m)}\right] \\
\text { s.t. } & \mathbb{E}\left[\sum_{m} w_{k, m}(\boldsymbol{\gamma}) \hat{r}^{(m)} \operatorname{PSR}_{k}^{(m)}\right] \geq \lambda_{k}, \forall k \in\left[1, N_{s}\right] \\
& \mathbf{1}^{\mathrm{T}} \mathbf{W}(\boldsymbol{\gamma}) \mathbf{1}=1, \forall \boldsymbol{\gamma} \in \mathbb{R}^{N_{s}} \\
& w_{k, m}(\boldsymbol{\gamma}) \geq 0, \forall \boldsymbol{\gamma} \in \mathbb{R}^{N_{s}}, k \in\left[1, N_{s}\right], m \in\left[0, N_{m}\right]
\end{array}
$$

where $\hat{r}^{(m)}$ is the average transmission rate of PHY mode $m$ normalized over a time slot, i.e., bits per time slot and is given by

$$
\hat{r}^{(m)}=r^{(m)} \frac{\Delta t}{T_{p}} .
$$

\footnotetext{
${ }^{1} \mathbb{E}\left[F_{k}(t)\right]>\lambda_{k}$ is referred to as strict stability.
} 
Substituting (4), (7), and (19) it can be written as

$$
\begin{array}{ll}
\text { Minimize: } & \mathbb{E}\left[\sum_{k} \sum_{m} w_{k, m}(\gamma) N_{p}^{(m)} E_{T P P}\right] \\
\text { s.t. } & \mathbb{E}\left[\sum_{m} w_{k, m}(\gamma) \frac{\mathrm{PSR}_{k}^{(m)}}{N_{p}^{(m)}} \frac{\Delta t}{T_{p}}\right] \geq \lambda_{k}, \quad \forall k \in\left[1, N_{s}\right] \\
& \mathbf{1}^{\mathrm{T}} \mathbf{W}(\gamma) \mathbf{1}=1, \forall \gamma \in \mathbb{R}^{N_{s}} \\
& w_{k, m}(\gamma) \geq 0, \forall \gamma \in \mathbb{R}^{N_{s}}, k \in\left[1, N_{s}\right], m \in\left[0, N_{m}\right]
\end{array}
$$

We denote the term $\mathrm{PSR}_{k}^{(m)} / N_{p}^{(m)}$ by $\chi_{k}^{(m)}$ which is referred to as the "effective rate" of the PHY mode $m$ and is a function of the channel state $\gamma_{k}$. Let $\mathbf{n}=\left(0, N_{p}^{(1)}, \ldots, N_{p}^{\left(N_{m}\right)}\right)^{\mathrm{T}}$, $\boldsymbol{\lambda}=\left(\lambda_{1}, \ldots, \lambda_{k}\right)^{\mathrm{T}}, \chi_{k}=\left(0, \chi_{k}^{(1)}, \ldots, \chi_{k}^{(m)}\right)^{\mathrm{T}}$, and $\boldsymbol{Z}^{\mathrm{T}}(\gamma)=$ $\left(\chi_{1}, \ldots, \chi_{N_{s}}\right)$. The stability policy is the solution of the following problem

Minimize: $\mathbb{E}\left[\mathbf{1}^{\mathrm{T}} \mathbf{W}(\boldsymbol{\gamma}) \mathbf{n}\right]$

s.t. $\quad \mathbb{E}[\mathbf{W}(\boldsymbol{\gamma}) \circ \boldsymbol{Z}(\boldsymbol{\gamma}) \mathbf{1}] \geq \frac{T_{p}}{\Delta t} \boldsymbol{\lambda}, \forall k \in\left[1, N_{s}\right]$

$$
\begin{aligned}
& \mathbf{1}^{\mathrm{T}} \mathbf{W}(\boldsymbol{\gamma}) \mathbf{1}=1, \forall \boldsymbol{\gamma} \in \mathbb{R}^{N_{s}} \\
& w_{k, m}(\boldsymbol{\gamma}) \geq 0, \forall \boldsymbol{\gamma} \in \mathbb{R}^{N_{s}}, k \in\left[1, N_{s}\right], m \in\left[0, N_{m}\right]
\end{aligned}
$$

where $\circ$ denotes the Hadamard (element by element) product and the total energy is normalized. To solve this problem, the channel state space should be properly discretized to a sufficiently large number of states. In this case, the scheduling policy maps the SNR partitions into a set of PHY mode probabilities for each sensor node. Nevertheless, it follows that the number of optimization variables grows exponentially with the network size. An alternative approach that can simplify the problem would be the case when the stability constraint is satisfied for each channel realization (and hence for each time slot). In this case, the scheduling policy selects with probability one the minimum-energy PHY mode with an instantaneous effective rate greater than or equal to the expected arrival rate of the sensor. Explicitly, we have

$$
w_{k, m}(\gamma)=\left\{\begin{array}{lc}
1, & \text { if } \chi_{k}^{(m)} \geq \frac{T_{p}}{\Delta t} \lambda_{k} \text { and } N_{p}^{(m)} \leq N_{p}^{(j)}, \forall j, k \\
0, & \text { otherwise. }
\end{array}\right.
$$

The minimum energy function, which is defined as the minimum average energy per symbol to stabilize the system with a given arrival rate vector $\lambda$ and SNR $\gamma$ can be expressed as

$$
\Phi(\boldsymbol{\lambda}, \gamma)=\mathbb{E}\left[\mathbf{1}^{\mathrm{T}} \mathbf{W}_{\boldsymbol{\lambda}}(\boldsymbol{\gamma}) \mathbf{n}\right]
$$

where $\mathbf{W}_{\boldsymbol{\lambda}}(\gamma)$ is the scheduling policy corresponding the given arrival rate vector $\lambda$ and channel state vector $\gamma$. Since $\chi_{k}^{(m)} \in[0,1] \forall k, m$, we limit the maximum possible arrival rate so that $\lambda_{k} \leq \lambda_{k, \max }=\frac{\Delta t}{T_{p}}, \forall k$ and discard the other scenarios. Clearly, for all $k$ if $\lambda_{k}>\lambda_{k, \max }$, then we have $\lambda_{k} \in \bar{\Lambda}$.

\section{Minimum Energy Subject to Stability With CHANNEl AND QueUE LENGTH INFORMATION}

The scheduling policy presented in the previous section is based on the average received SNR from the sensors as well as their average arrival rates. Due to the inherent difficulties in solving (21) and its need for the knowledge of average arrival rates, it is useful to pursue approaches based on the
QLI of the sensors, instead. Indeed, it is known that the scheduling policies that are only based on the channel state and ignore the queue backlog are sub-optimal [2]. The theory of Lyapunov drift seeks scheduling policies based on the queue and channel state information to stabilize the queuing systems. By incorporating a resource optimization part to this theory, it extends to Lyapunov optimization theory, which is extensively studied in the literature [2], [3], [5].

We apply the dynamic scheduling algorithm proposed in [2] and [4] to our IR-UWB network. The main idea is to exploit a specific function, i.e. a Lyapunov function that provides a numerical for the current stability level of the system based on its current state. Typically, the function is defined to grow large when the queue backlog of at least one sensor is approaching large values. We refer to the change in the Lyapunov function from one slot to another by the Lyapunov drift. It follows that the scheduling policy can stabilize the queuing system by taking control actions (i.e. allocating the PHY modes to the sensors) that make the Lyapunov drift in the negative direction towards zero. In general, the scheduling policy tries to minimize a drift-pluspenalty function which is sum of the Lyapunov drift and a weighted version of the required spectrum or energy resources that leads to minimum resource utilization while stabilizing the system. We are particularly minimizing the total required energy. Hence, the penalty weight can help the scheduling policy in order to put the system close to any desired point on the optimal energy-delay tradeoff curve.

Given the positive control parameter $V$, the scheduling policy is based on the QLI and CSI of all sensor nodes and the following parameters

$$
\begin{aligned}
\epsilon & =\frac{1}{\sqrt{(V)}}, \\
\omega & =\frac{\epsilon}{\delta_{\max }^{2}} e^{-\epsilon / \delta_{\max }}, \\
Q_{t h} & =\frac{6}{\omega} \ln \left(\frac{1}{\epsilon}\right),
\end{aligned}
$$

where $\delta_{\max }=\max \left\{A_{\max }, \max _{\left(m=0, \ldots, N_{m}\right)}\left\{r^{(m)}\right\}\right\}$ is the maximum variation of the queue length, $\epsilon$ is the backlog drift, $Q_{t h}$ is the buffer partitioning threshold [2], and $\ln (\cdot)$ denotes natural logarithm. At each time slot $t$, the hub schedules the sensor $K(t)$ to transmit using the PHY mode $M_{k}(t)$ by solving the following optimization problem

$$
\left(M_{k}(t), K(t)\right)=\underset{k \in\left[0, N_{s}\right], m \in\left[0, N_{m}\right]}{\arg \min }\left\{V N_{p}^{(m)}-\tilde{W}_{k}(t) \chi_{k}^{(m)}\right\},
$$

where $\tilde{W}_{k}(t)=\max \left\{W_{k}(t), 0\right\}$ and

$$
\begin{aligned}
W_{k}(t) & =\mathrm{I}\left(Q_{k}(t) \geq Q_{t h}\right) \omega e^{\omega\left(Q_{k}(t)-Q_{t h}\right)} \\
& -\mathrm{I}\left(Q_{k}(t)<Q_{t h}\right) \omega e^{-\omega\left(Q_{k}(t)-Q_{t h}\right)}+2 X_{k}(t) .
\end{aligned}
$$

The hub then updates the auxiliary queue $X_{k}(t)$ such that

$$
\begin{gathered}
X_{k}(t+1)=\max \left\{X_{k}(t)-\chi_{k}^{\left(M_{k}(t)\right)} \mathrm{I}(K(t)=k)\right. \\
\left.-\epsilon \mathrm{I}\left(Q_{k}(t)<Q_{t h}\right), 0\right\} \\
+A_{k}(t)+\epsilon \mathrm{I}\left(Q_{k}(t) \geq Q_{t h}\right),
\end{gathered}
$$


TABLE I. IEEE 802.15.6 PHY MODES

\begin{tabular}{c|c|c|c}
$\mathrm{m}$ & Symbol time $T_{s}^{(m)}(\mathrm{ns})$ & $N_{P}^{(m)}$ & Bit rate $(\mathrm{Mbps})$ \\
\hline 1 & 2051.300 & 32 & 0.487 \\
2 & 1025.600 & 16 & 0.975 \\
3 & 512.820 & 8 & 1.950 \\
4 & 256.410 & 4 & 3.900 \\
5 & 128.210 & 2 & 7.800 \\
6 & 64.103 & 1 & 15.600
\end{tabular}

where $X_{k}(0)=0$ and $\mathrm{I}(\cdot)$ is the indicator function defined as

$$
\mathrm{I}(\text { condition })=\left\{\begin{array}{lr}
1, & \text { if } \text { condition is true } \\
0, & \text { otherwise }
\end{array}\right.
$$

The average delay of the system is $\frac{1}{N_{s}} \sum_{k=1}^{N_{s}} \bar{Q}_{k}$, where

$$
\bar{Q}_{k}=\lim _{t \rightarrow \infty} \frac{1}{t} \sum_{\tau=0}^{t-1} \mathbb{E}\left\{Q_{k}(\tau)\right\}
$$

is the average delay corresponding to the $k^{\prime}$ th sensor node by Little's theorem [9].

\section{CASe Study: IEEE 802.15.6}

As an example, we apply our theoretical framework to the IR-UWB physical layer of the IEEE.802.15.6 standard [7]. The mandatory PHY in this standard consists of six different data rates that are demonstrated in Table I. The success probabilities corresponding to the SHR, PHR, and PSDU packets are derived in [10] and can be calculated based on the bit error equation given by (8).

We use the channel model in [8] to simulate the multipath and exploit average channel statistics to quantify the channel quality. The mean $\mu$ value for this channel averaged over 1000 realizations is $\bar{\mu}_{t}=0.6181$. The sensor subscript $k$ is removed for a specified sensor for simplicity. This also applies to $\lambda$ in the rest of the paper. Fig.2 demonstrates $\Phi(\lambda, \bar{\gamma})$ in (23) for a single node, assuming different values of the arrival rate $\lambda$ and average SNR $\bar{\gamma}$ along with the corresponding optimal PHY mode given by (22). The infeasible region is labeled by $\bar{\Lambda}$ in which no stability policy can be found. It can be observed that $\Phi(\lambda, \bar{\gamma})$ is a decreasing function of $\bar{\gamma}$ for a fixed $\lambda$, since the high SNR regime can achieve improved effective rates with a lower number of pulses per symbol.

In the sequel we describe the simulations of an IEEE 802.15.6 IR-UWB WBAN consisting of two nodes and a hub with rate scheduling based on CSI and QLI at the hub. To assess the scheduling policy we consider different traffic scenarios and different PHY parameters. The CSI and QLI of all nodes are assumed to be known at the hub. The QLI of a sensor node is informed to the hub in each beacon interval and the CSIs can be blindly estimated by the hub. The scheduling algorithm selects a sensor node and the corresponding optimal PHY mode every beacon period and informs it to the sensors on the downlink channels.

The instantaneous channel at each time slot is simulated based on the model given in [8] and the corresponding multipath factor $\mu$ is calculated by integrating the square of the instantaneous channel's impulse response. We assume $B=1$ $\mathrm{GHz}, \Delta t / T_{p}=500$, and data arrival is Poisson distributed

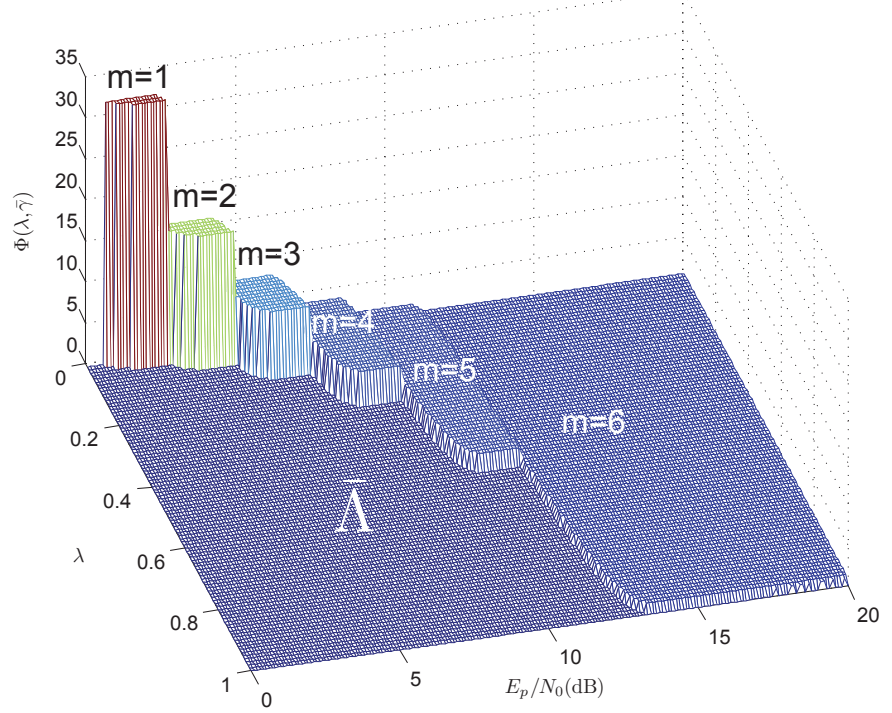

Fig. 2. Minimum energy function $\Phi(\lambda, \bar{\gamma})$ corresponding to the default IRUWB PHY option of the IEEE 802.15.6 standard with $\Delta t=T_{p}$. The required energy and the PHY mode to stabilize a queuing system with arrival rate $\lambda$ for a single node are given in the figure. The infeasible region is labeled by $\bar{\Lambda}$.

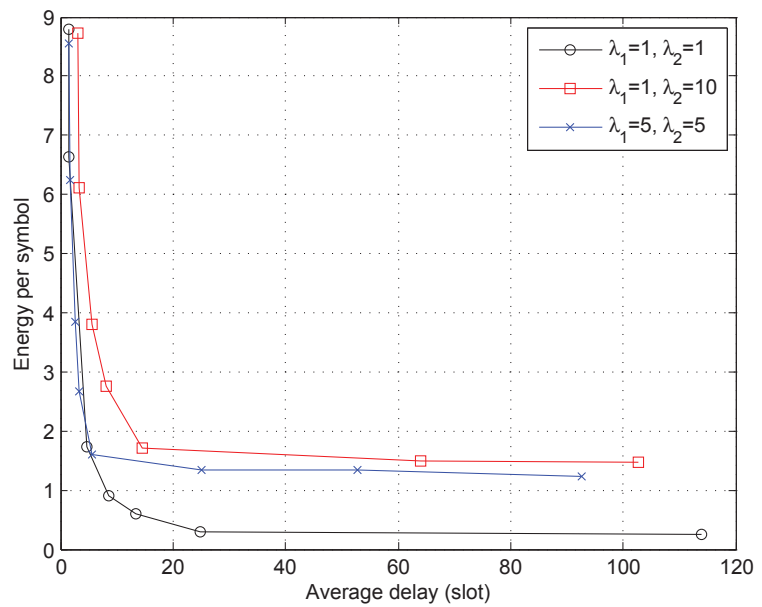

Fig. 3. The energy-delay tradeoff of the system considering different traffic scenarios.

which means the number of arrived data bits for sensor $k$ the time slot $t$ can be given by

$$
\mathbb{P}\left[A_{k}(t)=a\right]=\frac{\lambda^{a}}{a !} e^{-\lambda} .
$$

Each sensor node can either transmit using the PHY modes listed in Table I or wait in the idle mode which is indicated by $m=0$. The initial queue length is assumed zero for all sensors. We are firstly interested in the optimal energy-delay tradeoff in the system which can be achieved by varying the value of $V$ in (27). The simulations are performed for three different traffic scenarios and the resulting energy-delay tradeoff curves are depicted in Fig. 3. These tradeoffs are of significance importance in the design of the scheduling algorithm since they provide the explicit relationship between the average delay incurred by the queuing system at the sensors and the minimum amount of required energy to achieve it. We assume 


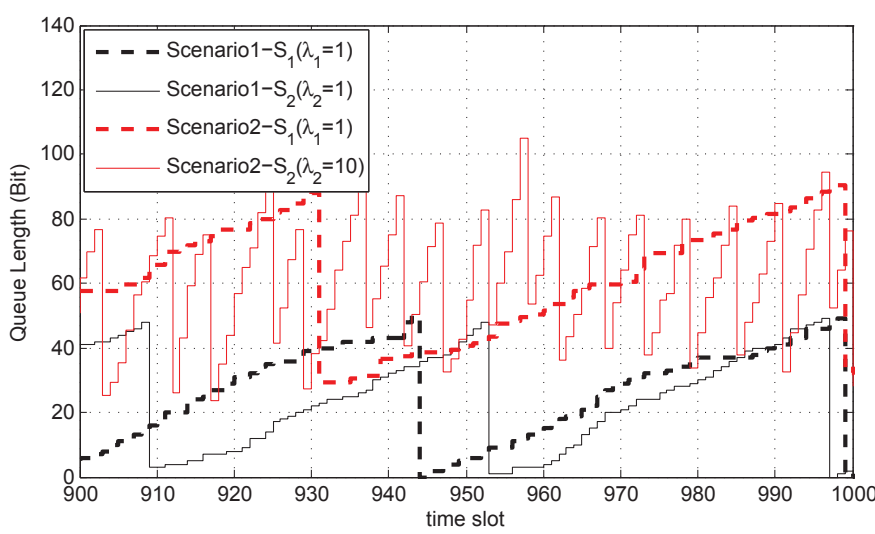

Fig. 4. Buffer evolution for different traffic arrival rates (equal average delay is assumed for the two scenarios).
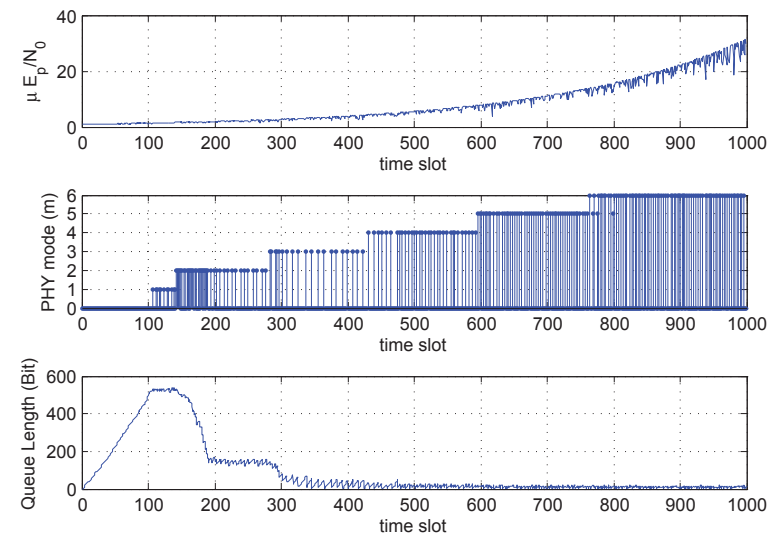

(a) sensor $s_{1}\left(\lambda_{1}=5\right)$
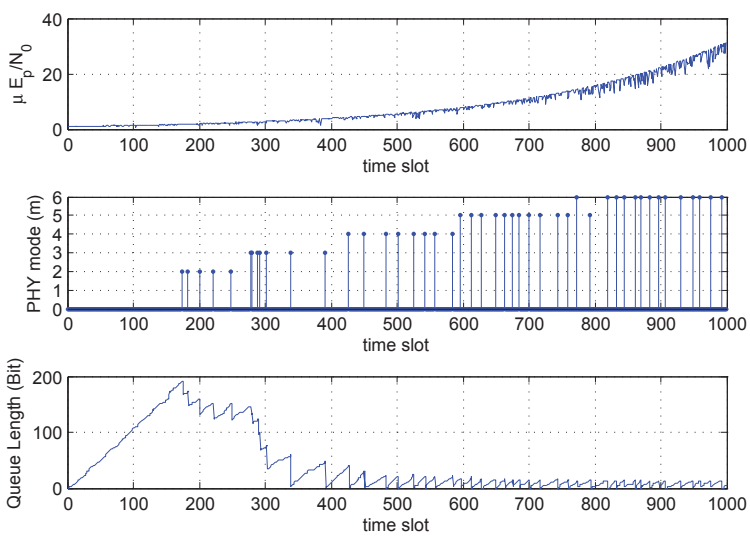

(b) sensor $s_{2}\left(\lambda_{2}=1\right)$

Fig. 5. Buffer evolution and rate scheduling with respect to the instantaneous channel state $\mu E_{p} / N_{0}$ (linear scale).

two scenarios with homogeneous arrivals $\lambda_{1}=\lambda_{2}=1 \mathrm{bit} /$ time slot and $\lambda_{1}=\lambda_{2}=5$ bits/time slot and a heterogeneous traffic scenario with $\lambda_{1}=1$ and $\lambda_{2}=10$. The average incurred delay for each node is calculated according to (31).

The steady state behavior of the sensor queues is depicted in Fig. 4 for various arrival rates. As a rule of thumb, the scheduling algorithm allocates the time slots more often to the sensors with higher arrival rates as long as they are in good channel conditions and the allocated PHY mode is more governed by the state of the channel. This idea is validated when we consider the state of the channel and observe the queue length together with the scheduled PHY mode which is illustrated in Fig. 5. In these figures we consider two sensors and different arrival rates $\lambda_{1}=5$ and $\lambda_{2}=1$. The value of $E_{p} / N_{0}$ is equal to zero at the first time slot and is increased by $0.015 \mathrm{~dB}$ so that we could study its impact on the selected PHY mode and the steady state conditions of the buffers. The state of channel can be assessed by observing the instantaneous value of $\mu E_{p} / N_{0}$ at each time slot. It can be seen that when the channel is not in a good condition (low $\mu E_{p} / N_{0}$ ) none of the sensors are selected by the scheduling algorithm and both queue lengths increase linearly (constant arrival and zero service rate). By increasing $\mu E_{p} / N_{0}$, the queue backlogs are stabilized after it reaches a specific point and tend to zero as it increases further. It can also be observed that the higher PHY modes are allocated in better channel conditions which is expected a priori. The scheduling algorithm can be tuned by changing the $V$ parameter to approach any desired point in the optimal energy-delay tradeoff curve.

\section{CONCLUSION}

We studied the energy-delay tradeoffs in IR-UWB WBANs with noncoherent receivers and applied this framework to the IEEE 802.15.6 networks. The stability scheduling policies based on CSI and QLI were addressed and simulated using the standard PDP model of the IEEE 802.15.6 channel model. We also analyzed the dynamic behavior of the rate scheduling policy in different traffic and channel conditions and showed that our rate scheduling policy can respond to these variations in order to preserve the stability with an energy arbitrary close to the minimum possible energy.

\section{REFERENCES}

[1] B. Latre, B. Braem, I. Moennan, C. Blondia, and P. Demeester, "A survey on wireless body area networks," Journal of Wireless Networks, vol. 17, pp. 1-18, 2011.

[2] M. Neely, "Optimal energy and delay tradeoffs for multiuser wireless downlinks," IEEE Transactions on Information Theory, vol. 53, no. 9, pp. 30953113, Sep. 2007.

[3] M. Neely, "Energy optimal control for time-varying wireless networks," IEEE Transactions on Information Theory, vol. 52, no. 7, pp. 29152934, July 2006.

[4] E. Rebeiz, G. Caire, A.F. Molisch, "Energy-delay tradeoff and dynamic sleep switching for bluetooth-like body-area sensor networks," IEEE Transactions on Communications, vol. 60, no. 9, pp. 2733-2746, Sep. 2012.

[5] L. Tassiulas, A. Ephremides, "Stability properties of constrained queueing systems and scheduling policies for maximum throughput in multihop radio networks,' IEEE Transactions on Automatic Control, vol.37, no.12, pp.1936,1948, Dec 1992.

[6] M. S. Mohammadi, Q. Zhang, E. Dutkiewicz, X, Huang, "Optimal energy efficiency link adaptation in IEEE 802.15.6 IR-UWB wireless body area networks," Submitted to IEEE Communications Letters.

[7] "IEEE Standard for local and metropolitan area networks - part 15.6: wireless body area networks," IEEE Std 802.15.6-2012, 2012.

[8] IEEE 802.15 WPAN task group 6 (TG6) body area networks. [Online]. Available: http://www.ieee802.org/15/pub/TG6.html

[9] S. Asmussen, "Applied probability and queues", John Wiley and Sons, N.Y., 1987.

[10] M. S. Mohammadi, Q. Zhang, E. Dutkiewicz, X. Huang, "Optimal frame length to maximize energy efficiency in IEEE 802.15.6 UWB body area networks," IEEE Wireless Communications Letters, 2014. 